\title{
ON NON-CONTRACTIBLE PERIODIC ORBITS OF HAMILTONIAN DIFFEOMORPHISMS
}

\author{
BAŞAK Z. GÜREL
}

\begin{abstract}
We prove that any Hamiltonian diffeomorphism of a closed symplectic manifold equipped with an atoroidal symplectic form has simple noncontractible periodic orbits of arbitrarily large period, provided that the diffeomorphism has a non-degenerate (or even isolated and homologically nontrivial) periodic orbit with non-zero homology class and the set of one-periodic orbits in that class is finite.
\end{abstract}

\section{CONTEnTs}

1. Introduction and main results 1

1.1. Acknowledgements 3

2. Preliminaries 4

2.1. Conventions and notation 4

2.2. Floer homology for non-contractible periodic orbits 4

3. Proof of Theorem $1.1 \quad 5$

$\begin{array}{ll}\text { References } & 7\end{array}$

\section{INTRODUCTION AND MAIN RESULTS}

In this paper, we study the question of existence of non-contractible periodic orbits for Hamiltonian diffeomorphisms $\varphi_{H}$ of a closed symplectic manifold $\left(M^{2 n}, \omega\right)$ such that the integral of $\omega$ over all toroidal classes in $\mathrm{H}_{2}(M ; \mathbb{Z})$ vanishes. Under this condition, we prove that $\varphi_{H}$ has non-contractible periodic orbits of arbitrarily large prime period, provided that it has one non-degenerate (or isolated and homologically non-trivial) periodic orbit with homology class non-zero modulo torsion.

Non-contractible periodic orbits of Hamiltonian systems have been previously investigated in a number of papers using Floer theoretical techniques; see, e.g., [BPS, BuHal, GaL, Lee, Ni, We]. The central theme of this paper, however, lies in a different domain. Conceptually, the key difference is that here we allow the period of the orbits to vary, whereas the above works concern the existence of periodic orbits (in a given free homotopy class) of a fixed period. In other words, we approach the problem from a dynamical systems perspective rather than topological. On the technical side, the source of periodic orbits in those works is non-vanishing Floer homology or other more subtle invariants such as Floer-theoretic torsion. In general,

Date: April 4, 2018.

2000 Mathematics Subject Classification. 53D40, 37J10.

Key words and phrases. Non-contractible periodic orbits, Hamiltonian flows, Floer homology.

The work is partially supported by the NSF grants DMS-0906204 and DMS-1207680. 
one major obstacle to employing this method in the investigation of non-contractible periodic orbits for closed manifolds is that the total non-contractible Floer homology is zero since all one-periodic orbits of a $C^{2}$-small autonomous Hamiltonian are constant, and hence contractible. (Thus even the entire Floer complex may be zero.) Therefore, the previous results mainly apply to open manifolds such as ordinary and twisted cotangent bundles; see, e.g., [BPS, Ni, We]. Working with closed manifolds, we take a different approach. In our case, the source for periodic orbits is the change in certain filtered Floer homology groups under the iteration of the Hamiltonian, and the original non-contractible orbit spawns infinitely many other orbits. (Our argument shares many common elements with that [Gü].)

To put our result in perspective in a different way, recall the following variant of the Conley conjecture, referred to as the HZ-conjecture in [Gü]. This is the assertion that a Hamiltonian diffeomorphism with "more than necessary" non-degenerate (or just homologically non-trivial) fixed points has infinitely many periodic orbits. Here "more than necessary" is usually interpreted as a lower bound arising from some version of the Arnold conjecture. For $\mathbb{C P}^{n}$, the expected threshold is $n+1$. This conjecture, originally stated in [HZ], is inspired by a celebrated theorem of Franks stating that a Hamiltonian diffeomorphism (or, even, an area preserving homeomorphism) of $S^{2}$ with at least three fixed points must have infinitely many periodic orbits, [Fr1, Fr2]; see also [FH, LeC] for further refinements and [BraHo, CKRTZ, Ker] for symplectic topological proofs.

Viewing the HZ-conjecture in a broader context is often useful. Namely, it appears that the presence of a fixed point that is unnecessary from a homological or geometrical perspective is already sufficient to force the existence of infinitely many periodic orbits. For instance, a theorem from [GG4] asserts that, for a certain class of closed monotone symplectic manifolds including $\mathbb{C P}^{n}$, any Hamiltonian diffeomorphism with a hyperbolic fixed point must necessarily have infinitely many periodic orbits; see also [Gü] for other results along these lines. (Note also that these considerations are related to and motivated by the circle of questions in the realm of the original Conley conjecture; see, [FH, Gi2, GG3, Hi1, LeC, SZ] and also [CGG, GG1, He] for a detailed account of this conjecture and related results.)

The main result of the paper therefore can be viewed as the proof of the generalized HZ-conjecture for non-contractible periodic orbits of Hamiltonian diffeomorphisms. Indeed, observe that from a homological perspective a Hamiltonian diffeomorphism of a closed symplectic manifold need not have a non-contractible periodic orbit. However, our result shows that, if such an orbit exists, it generates infinitely many others.

Let us now state the main result of the paper. Let $\left(M^{2 n}, \omega\right)$ be a closed symplectic manifold equipped with an atoroidal symplectic form $\omega$. (Namely, we assume that for every map $v$ : $\mathbb{T}^{2} \rightarrow M$, the integral of $\omega$ over $v$ vanishes, i.e., $\langle[\omega],[v]\rangle=0$.) For a Hamiltonian diffeomorphism $\varphi_{H}$ of $M$, denote by $\mathcal{P}_{1}\left(\varphi_{H},[\gamma]\right)$ the collection of one-periodic orbits of $\varphi_{H}$ with homology class $[\gamma] \in \mathrm{H}_{1}(M ; \mathbb{Z}) /$ Tor.

Theorem 1.1. Let $M$ be a closed symplectic manifold equipped with an atoroidal symplectic form $\omega$. Assume that a Hamiltonian diffeomorphism $\varphi_{H}$ of $M$ has a non-degenerate one-periodic orbit $\gamma$ with homology class $[\gamma] \neq 0$ in $\mathrm{H}_{1}(M ; \mathbb{Z}) /$ Tor and that $\mathcal{P}_{1}\left(\varphi_{H},[\gamma]\right)$ is finite. Then, for every sufficiently large prime $p$, the diffeomorphism $\varphi_{H}$ has a simple periodic orbit with homology class $p[\gamma]$ and period either $p$ or $p^{\prime}$, where $p^{\prime}$ is the first prime number greater than $p$. 
Corollary 1.2. In the setting of Theorem 1.1 (or Theorem 3.1), the number of non-contractible periodic orbits with period less than or equal to $k$, or the number of distinct homology classes represented by such orbits, is bounded from below by const $\cdot k / \log k$.

An immediate consequence of Theorem 1.1 is that $\varphi_{H}$ has infinitely many periodic orbits with homology classes in $\mathbb{N}[\gamma]$ whether or not $\mathcal{P}_{1}\left(\varphi_{H},[\gamma]\right)$ is finite. Moreover, the non-degeneracy condition in the theorem can be relaxed and replaced by a much weaker, albeit more technical, requirement that $\gamma$ is isolated and homologically non-trivial, i.e., its local Floer homology is non-zero. (See Section 3 for a discussion of this notion.) Finally, the orbit $\gamma$ need not be one-periodic; the theorem (with obvious modifications) still holds when $\gamma$ is just a periodic orbit.

Among the manifolds meeting the requirements of the theorem are, for instance, all closed Kähler manifolds with negative sectional curvature (e.g., complex hyperbolic spaces) or, more generally, any closed symplectic manifold $M$ such that $\pi_{2}(M)=0$ and $\pi_{1}(M)$ is a hyperbolic group. Indeed, in this case any map $v: \mathbb{T}^{2} \rightarrow M$ is homologous to zero over $\mathbb{R}$, since $\pi_{1}(M)$ contains no subgroups isomorphic to $\mathbb{Z} \oplus \mathbb{Z}$ (see, e.g., [BriHae, GhdlH]), and hence the pull-back homomorphism

$$
v^{*}: \mathrm{H}^{2}(M ; \mathbb{R})=\mathrm{H}^{2}\left(K\left(\pi_{1}(M, 1) ; \mathbb{R}\right)\right) \rightarrow \mathrm{H}^{2}\left(\mathbb{T}^{2} ; \mathbb{R}\right)
$$

is necessarily zero. (See [BruK, Ked] and also references therein for other examples.)

The existence of a non-contractible periodic orbit $\gamma$ as in Theorem 1.1 appears to be a common occurrence. In fact, we are not aware of any example of a Hamiltonian diffeomorphism with isolated fixed points which obviously would not have a non-contractible periodic orbit, provided that $\mathrm{H}_{1}(M ; \mathbb{R}) \neq 0$. However, let us emphasize that the homology class of $\gamma$ cannot be prescribed in advance, for, as simple examples (e.g., the height function on $\mathbb{T}^{2}$ ) show, non-contractible orbits need not exist in a given homology class or in the multiples of a given class. (In fact, KAM theory implies that this is even generically the case, at least in dimension two.)

The growth rate established in Corollary 1.2 is typical in this class of questions in the absence of hyperbolicity. For instance, a similar growth rate is known to hold in the context of the Conley conjecture (see, e.g., [Gi2, GG1, He, Hi1, SZ]) and for closed geodesics on $S^{2}$; see, e.g., [Hi2]. (There are, however, stronger growth results; see, e.g., [LeC, Vi].) Perhaps it is also worth pointing out that Theorem 1.1 and Corollary 1.2 are reminiscent of some results on the growth of prime geodesics on negatively curved manifolds (with the scale exponentially adjusted); see, e.g., [AS, Ma, PS]. However, to the best our understanding, there is essentially no connection, technical or conceptual, between the respective results beyond a superficial resemblance.

Finally, the question of possible generalizations of Theorem 1.1 to the case when $M$ is toroidally monotone will be addressed elsewhere. (Recall that $M$ is said to be toroidally monotone if for every map $v: \mathbb{T}^{2} \rightarrow M$ we have $\langle[\omega],[v]\rangle=\lambda\left\langle c_{1}(T M),[v]\right\rangle$ for some constant $\lambda \geq 0$ independent of $v$. See [GG4] for a partial result in this direction.)

1.1. Acknowledgements. The author is grateful to Viktor Ginzburg, Denis Osin, and Ioana Şuvaina for useful discussions. 


\section{Preliminaries}

In this section, we set conventions and notation, and discuss Floer homology for non-contractible periodic orbits.

2.1. Conventions and notation. Throughout the paper, we assume that $\left(M^{2 n}, \omega\right)$ is a closed manifold equipped with an atoroidal symplectic form $\omega$, i.e., for every map $v: \mathbb{T}^{2} \rightarrow M$, the integral of $\omega$ over $v$ vanishes: $\langle[\omega],[v]\rangle=0$. We refer the reader to Section 1 for examples of such (open or closed) symplectic manifolds and here only note that they arise naturally as the simplest setting where Floer theory for non-contractible periodic orbits is defined.

All Hamiltonians $H$ are assumed to be one-periodic in time, i.e., $H: S^{1} \times M \rightarrow \mathbb{R}$, and we set $H_{t}=H(t, \cdot)$ for $t \in S^{1}=\mathbb{R} / \mathbb{Z}$. The Hamiltonian vector field $X_{H}$ of $H$ is defined by $i_{X_{H}} \omega=-d H$. The (time-dependent) flow of $X_{H}$ is denoted by $\varphi_{H}^{t}$ and its time-one map by $\varphi_{H}$. Such time-one maps are referred to as Hamiltonian diffeomorphisms. The Hofer norm of $H$ is $\|H\|=\int_{S^{1}}\left[\max _{M} H_{t}-\min _{M} H_{t}\right] d t$.

Let $K$ and $H$ be two one-periodic Hamiltonians. The composition $K \downarrow H$ is defined by the formula

$$
(K \downarrow H)_{t}=K_{t}+H_{t} \circ\left(\varphi_{K}^{t}\right)^{-1},
$$

and the flow of $K \natural H$ is $\varphi_{K}^{t} \circ \varphi_{H}^{t}$. We set $H^{\natural k}=H \natural \ldots \natural H$ ( $k$ times). Abusing terminology, we will refer to $H^{\natural k}$ as the $k$ th iteration of $H$. (Note that the flow $\varphi_{H^{\natural k}}^{t}=\left(\varphi_{H}^{t}\right)^{k}, t \in[0,1]$, is homotopic with fixed end-points to the flow $\varphi_{H}^{t}$, $t \in[0, k]$.

In general, $H^{\natural k}$ is not one-periodic, even when $H$ is. However, this is the case if, for example, $H_{0} \equiv 0 \equiv H_{1}$. The latter condition can be met by reparametrizing the Hamiltonian as a function of time without changing the time-one map; the Hofer norm, and actions and indices of the periodic orbits do not change during the procedure. Thus, in what follows, we always treat $H^{\natural k}$ as a one-periodic Hamiltonian.

The $k$ th iteration of a one-periodic orbit $\gamma$ of $H$ is denoted by $\gamma^{k}$. More specifically, $\gamma^{k}(t)=\varphi_{H \natural k}^{t}(\gamma(0))$, where $t \in[0,1]$. We can think of $\gamma^{k}$ as the $k$-periodic orbit $\gamma(t), t \in[0, k]$, of $H$. Hence, there is an action-preserving one-to-one correspondence between one-periodic orbits of $H^{\natural k}$ and $k$-periodic orbits of $H$.

2.2. Floer homology for non-contractible periodic orbits. The key tool used in the proof of Theorem 1.1 is the filtered Floer homology for non-contractible periodic orbits of Hamiltonian diffeomorphisms. Various flavors of Floer homology in this case for both open and closed manifolds have been considered in several other works; see, e.g., [BPS, BuHal, GaL, GG4, Lee, Ni, We]. Below we briefly describe the elements of the construction relevant to our case.

Let $\zeta$ be a free homotopy class of maps $S^{1} \rightarrow M$. Fix a reference loop $z \in \zeta$ and a symplectic trivialization of $T M$ along $z$. (In fact, it would be sufficient to fix a trivialization of the canonical bundle of $M$ along $z$.) A capping of $x: S^{1} \rightarrow M$ with free homotopy class $\llbracket x \rrbracket=\zeta$ is a cylinder (i.e., a homotopy) $\Pi:[0,1] \times S^{1} \rightarrow M$ connecting $x$ and $z$. The action of a one-periodic Hamiltonian $H$ on $x$ is

$$
\mathcal{A}_{H}(x)=-\int_{\Pi} \omega+\int_{S^{1}} H_{t}(x(t)) d t .
$$

The action $\mathcal{A}_{H}(x)$ is well-defined, i.e., independent of capping, for $\omega$ is atoroidal and hence $\langle[\omega],[v]\rangle=0$, where $v: \mathbb{T}^{2} \rightarrow M$ is the torus obtained by attaching two different cappings to each other. Moreover, the critical points of $\mathcal{A}_{H}$ are exactly 
one-periodic orbits of the time-dependent Hamiltonian flow $\varphi_{H}^{t}$ with homotopy class in $\zeta$. The action spectrum $\mathcal{S}(H, \zeta)$ is the set of critical values of $\mathcal{A}_{H}$. It has zero measure; see, e.g., [HZ].

Observe that the trivialization of $T M$ along the reference loop $z$ uniquely extends to a trivialization along $\Pi$, and hence induces a trivialization along $x$. Using this trivialization, the Conley-Zehnder index $\mu_{\mathrm{CZ}}(H, x) \in \mathbb{Z}$ of a non-degenerate orbit $x$ is defined as in [Sa, SZ]. (Here $x$ is said to be non-degenerate if the linearized return map $d \varphi_{H}: T_{x(0)} M \rightarrow T_{x(0)} M$ does not have one as an eigenvalue.) Similarly to the contractible case, the Conley-Zehnder index is defined only modulo $2 N$, where $N$ is the minimal "toroidal" Chern number of $M$, unless $c_{1}(T M)$ vanishes on toroidal homology classes in $\mathrm{H}_{2}(M ; \mathbb{Z})$. Note that, by definition, $N$ is the positive generator of the subgroup of $\mathbb{Z}$ generated by the integrals of $c_{1}(T M)$ over all toroidal classes.

The construction of Floer homology $\operatorname{HF}(H, \zeta)$ and the filtered Floer homology $\operatorname{HF}^{(a, b)}(H, \zeta)$, taking into account only the one-periodic orbits with homotopy classes in $\zeta$, goes through exactly as in the contractible case; see, e.g., [BPS, Lee].

Recall that the total Floer homology in a class $\zeta \neq 0$ is trivial: $\operatorname{HF}(H, \zeta)=0$; for all one-periodic orbits of a $C^{2}$-small autonomous Hamiltonian are constant, and hence contractible. However, the filtered Floer homology for some action interval is obviously non-zero whenever there exists an isolated and homologically non-trivial (e.g., non-degenerate) periodic orbit $\gamma \in \zeta$. Indeed, assuming for simplicity that $\mathcal{A}_{H}(\gamma)=0$, observe that $\mathrm{HF}^{(-a, a)}(H, \llbracket \gamma \rrbracket) \neq 0$, for instance, when $(-a, a)$ contains only the action value zero for $a \notin \mathcal{S}(H, \llbracket \gamma \rrbracket)$. This follows from the fact that

$$
\operatorname{HF}^{(-a, a)}(H, \llbracket \gamma \rrbracket)=\operatorname{HF}(\gamma) \oplus \ldots \neq 0,
$$

where $\operatorname{HF}(\gamma)$ is the local Floer homology of $H$ at $\gamma$ (see, e.g., [Fl1, Fl2, Gi2, GG2]), and the dots represent the local Floer homology contributions from other orbits in the class $\llbracket \gamma \rrbracket$ with action equal to zero; see, e.g., [GG2] for a proof of this fact.

The action and the index of periodic orbits (and hence the grading and filtration of the Floer complex) do depend on the choice of reference curves $z$ and, in the index case, on the trivialization of $T M$ along $z$. Whenever we consider the iteration $H^{\natural \kappa}$ of $H$, we simultaneously iterate each class $\zeta$ and the reference curve $z$ (i.e., we pass to $\zeta^{\kappa}$ and $z^{\kappa}$ ). As a consequence, the action functional on iterated $\kappa$-periodic orbits is homogeneous with respect to the iterations, i.e.,

$$
\mathcal{A}_{H_{\text {६ }}}\left(x^{\kappa}\right)=\kappa \mathcal{A}_{H}(x) .
$$

\section{Proof of Theorem 1.1}

As has been mentioned in the introduction, we establish a more general result. To state it, recall that an isolated periodic orbit $\gamma$ of $\varphi_{H}$ is said to be homologically non-trivial if the local Floer homology of $H$ at $\gamma$ is non-zero. For instance, a nondegenerate fixed point is homologically non-trivial. More generally, an isolated fixed point with non-vanishing topological index is homologically non-trivial; for this index is equal, up to a sign, to the Euler characteristic of the local Floer homology. The notion of homological non-triviality seems to be particularly wellsuited for use in the context of Conley conjecture-type questions; we refer the reader to [Gü] for a detailed discussion of this condition. Theorem 1.1 is an immediate consequence of the following result. 
Theorem 3.1. Let $M$ be a closed symplectic manifold equipped with an atoroidal symplectic form $\omega$. Assume that a Hamiltonian diffeomorphism $\varphi_{H}$ of $M$ has an isolated and homologically non-trivial one-periodic orbit $\gamma$ with homology class $[\gamma] \neq$ 0 in $\mathrm{H}_{1}(M ; \mathbb{Z}) /$ Tor and that $\mathcal{P}_{1}\left(\varphi_{H},[\gamma]\right)$ is finite. Then, for every sufficiently large prime $p$, the diffeomorphism $\varphi_{H}$ has a simple periodic orbit with homology class $p[\gamma]$ and period either $p$ or $p^{\prime}$, where $p^{\prime}$ is the first prime number greater than $p$.

Here, as in Section 1, $\mathcal{P}_{1}\left(\varphi_{H},[\gamma]\right)$ is the collection of one-periodic orbits of $\varphi_{H}$ with homology class $[\gamma]$ in $\mathrm{H}_{1}(M ; \mathbb{Z}) /$ Tor.

Remark 3.2. In fact, the homology class $p[\gamma]$ in the assertion of the theorem can be replaced by the free homotopy class $\llbracket \gamma \rrbracket^{p}:=\llbracket \gamma^{p} \rrbracket$, provided that $\llbracket \gamma \rrbracket \neq 0$ and, for instance, the only solutions to the equation $\llbracket \gamma \rrbracket^{p}=\zeta^{q}$, where $p$ and $q$ are sufficiently large primes and $\zeta$ is a free homotopy class, are $\zeta=\llbracket \gamma \rrbracket$ and $p=q$.

Proof of Theorem 3.1. First, recall that all sufficiently large prime numbers $p$ are admissible in the sense of [GG2]. Thus, under such iterations of $H$, the orbit $\gamma$ stays isolated, and

$$
\operatorname{HF}\left(\gamma^{p}\right)=\operatorname{HF}(\gamma)
$$

by the persistence of local Floer homology theorem in [GG2]. In particular, in our case, $\operatorname{HF}\left(\gamma^{p}\right) \neq 0$ since $\operatorname{HF}(\gamma) \neq 0$. From now on, we work with primes that are large enough and hence admissible, which we order as $p_{1}<p_{2}<\ldots$. In what follows, $p$ or $p_{i}$ always denotes a prime from this sequence.

Proceeding with the proof, assume that $p_{i}$ is a sufficiently large prime number such that $\varphi_{H}$ has no simple $p_{i}$-periodic orbit in the homology class $p_{i}[\gamma]$. (If such a prime does not exist then we are done.) Our goal is to show that in this case $\varphi_{H}$ must have a simple $p_{i+1}$-periodic orbit in the homology class $p_{i}[\gamma]$.

Let $\gamma_{1}=\gamma, \gamma_{2}, \ldots, \gamma_{r}$ be the set of one-periodic orbits of $\varphi_{H}$ such that $\left[\gamma_{l}\right]=[\gamma]$ for $l=1, \ldots, r$. Due to the above assumption, any $p_{i}$-periodic orbit of $\varphi_{H}$ with homology class $p_{i}[\gamma]$ is the $p_{i}$ th iteration of some one-periodic orbit $\beta$ of $\varphi_{H}$. On the other hand, clearly $\left[\beta^{p_{i}}\right]=p_{i}[\gamma]$ if and only if $[\beta]=[\gamma]$. Thus $\beta$ must be one of the orbits $\gamma_{l}$. In particular,

$$
\mathcal{S}\left(H^{\natural p_{i}}, \llbracket \gamma \rrbracket^{p_{i}}\right) \subset p_{i} \bigcup_{l=1}^{r} \mathcal{S}\left(H, \llbracket \gamma_{l} \rrbracket\right),
$$

where, as in Section 2, $\llbracket \gamma \rrbracket$ is the free homotopy class of $\gamma$ and $\llbracket \gamma \rrbracket^{p_{i}}:=\llbracket \gamma^{p_{i}} \rrbracket$.

Assume without loss of generality that $\mathcal{A}_{H}(\gamma)=0$, which we can ensure by adding a constant to $H$; hence $\mathcal{A}_{H^{\natural p}}\left(\gamma^{p}\right)=0$ for all iterations $p$ by (2.1). Following closely [Gü], pick $a>0$ outside $\bigcup_{l=1}^{r} \mathcal{S}\left(H, \llbracket \gamma_{l} \rrbracket\right)$ such that 0 is the only action value in $(-a, a)$. Therefore, 0 is the only point of $\mathcal{S}\left(H^{\natural p_{i}}, \llbracket \gamma \rrbracket^{p_{i}}\right)$ in the interval $\left(-p_{i} a, p_{i} a\right)$, and hence

$$
\operatorname{HF}^{\left(-p_{i} a, p_{i} a\right)}\left(H^{\natural p_{i}}, \llbracket \gamma \rrbracket^{p_{i}}\right)=\operatorname{HF}\left(\gamma^{p_{i}}\right) \oplus \ldots,
$$

where the dots represent the local Floer homology contributions from the remaining one-periodic orbits $\gamma_{l}$ with zero action such that $\llbracket \gamma_{l} \rrbracket^{p_{i}}=\llbracket \gamma \rrbracket^{p_{i}}$.

Let $C=\|H\|$ and choose $p_{i}$ so large that $p_{i} a>6 C\left(p_{i+1}-p_{i}\right)$. The latter is guaranteed by the fact that $p_{i+1}-p_{i}=o\left(p_{i}\right)$; see, e.g., [BHP]. Now, pick $\alpha>0$ such that $p_{i} a-4 C\left(p_{i+1}-p_{i}\right)<\alpha<p_{i} a-2 C\left(p_{i+1}-p_{i}\right)$. Then we have

$$
-p_{i} a<-\alpha<-\alpha+2 C\left(p_{i+1}-p_{i}\right)<0<\alpha<\alpha+2 C\left(p_{i+1}-p_{i}\right)<p_{i} a,
$$


and also

$$
-p_{i+1} a<-\alpha+C\left(p_{i+1}-p_{i}\right)<0<\alpha+C\left(p_{i+1}-p_{i}\right)<p_{i+1} a .
$$

Set $\delta_{i}:=C\left(p_{i+1}-p_{i}\right)$ and denote by $(a, b)+\epsilon$ the shifted interval $(a+\epsilon, b+\epsilon)$. For a linear homotopy from $H^{\natural p_{i}}$ to $H^{\natural p_{i+1}}$, we have the induced map

$$
\mathrm{HF}^{(-\alpha, \alpha)}\left(H^{\natural p_{i}}, \llbracket \gamma \rrbracket^{p_{i}}\right) \rightarrow \mathrm{HF}^{(-\alpha, \alpha)+\delta_{i}}\left(H^{\natural p_{i+1}}, \llbracket \gamma \rrbracket^{p_{i}}\right)
$$

in filtered Floer homology. A proof of this fact for the ordinary filtered Floer homology can be found in [Gil]. This is also true in our case since continuation maps preserve homotopy classes of periodic orbits. Likewise, the linear homotopy from $H^{\natural p_{i+1}}$ to $H^{\natural p_{i}}$ results in another action shift in $\delta_{i}$. Consider now the following commutative diagram:

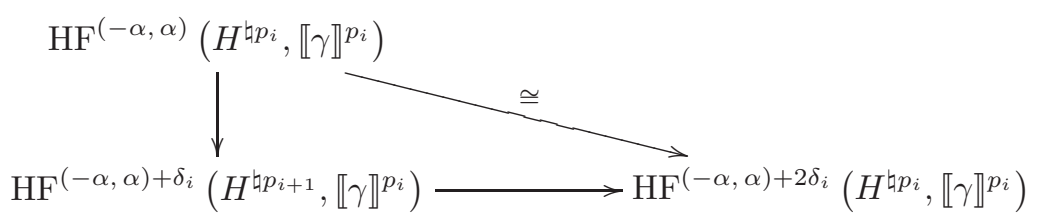

Here the filtered Floer homology groups of $H^{\natural p_{i}}$ are non-zero by (3.1) and the choice of $\alpha$. Furthermore, the diagonal map is an isomorphism induced by the natural quotient-inclusion map; see, e.g., [Gi1]. Indeed, zero is the only action value in the intervals $(-\alpha, \alpha)$ and $(-\alpha, \alpha)+2 \delta_{i}$, and the map is an isomorphism by the stability of the filtered Floer homology; see, e.g., [GG2].

We now have a non-zero isomorphism factoring through the filtered homology of $H^{\natural p_{i+1}}$ in the commutative diagram. In particular, this group cannot be zero:

$$
\mathrm{HF}^{(-\alpha, \alpha)+\delta_{i}}\left(H^{\natural p_{i+1}}, \llbracket \gamma \rrbracket^{p_{i}}\right) \neq 0 .
$$

Thus $\varphi_{H}$ must have a $p_{i+1}$-periodic orbit $\eta$ in the homotopy class $\llbracket \gamma \rrbracket^{p_{i}}$, and hence in the homology class $p_{i}[\gamma]$. What remains to show is that $\eta$ is necessarily simple, provided that $p_{i+1}$ (i.e., $p_{i}$ ) is large enough. Arguing by contradiction, assume that $\eta=\nu^{p_{i+1}}$ for some one-periodic orbit $\nu$. Then

$$
p_{i}[\gamma]=[\eta]=p_{i+1}[\nu] .
$$

Let us write $[\gamma]=n \sigma$ and $[\nu]=m \rho$ for some prime homology classes $\sigma$ and $\rho$ in $\mathrm{H}_{1}(M ; \mathbb{Z}) /$ Tor, where $n, m \in \mathbb{N}$. Using (3.2), we now see that $\sigma=\rho$ and $n p_{i}=m p_{i+1}$, where $n$ is fixed - it is determined by $\gamma$. This is clearly impossible once $p_{i+1}$ is greater than $n$.

\section{REFERENCES}

[AS] T. Adachi, T. Sunada, Homology of closed geodesics in a negatively curved manifold, J. Differential Geom., 26 (1987), 81-99.

[BHP] R.C. Baker, G. Harman J. Pintz, The difference between consecutive primes, II, Proc. London Math. Soc., 83 (2001), 532-562.

[BPS] P. Biran, L. Polterovich, D. Salamon, Propagation in Hamiltonian dynamics and relative symplectic homology, Duke Math. J., 119 (2003), 65-118.

[BraHo] B. Bramham, H. Hofer, First steps towards a symplectic dynamics, Preprint 2011, arXiv:1102.3723.

[BriHae] M. Bridson, A. Haefliger, Metric spaces of non-positive curvature, Springer-Verlag, Berlin, 1999. 
[BruK] M. Brunnbauer, D. Kotschick, On hyperbolic cohomology classes, Preprint 2008, arXiv:0808.1482.

[BuHal] D. Burghelea, S. Haller, Non-contractible periodic trajectories of symplectic vector fields, Floer cohomology and symplectic torsion, Preprint 2001, math.SG/0104013.

[CGG] M. Chance, V.L. Ginzburg, B.Z. Gürel, Action-index relations for perfect Hamiltonian diffeomorphisms, Preprint 2011, arXiv:1110.6728; to appear in J. Sympl. Geom.

[CKRTZ] B. Collier, E. Kerman, B.M. Reiniger, B. Turmunkh, A. Zimmer, A symplectic proof of a theorem of Franks, Preprint 2011, arXiv:1107.1282; to appear in Compos. Math.

[Fl1] A. Floer, Witten's complex and infinite-dimensional Morse theory, J. Differential Geom., 30 (1989), 207-221.

[Fl2] A. Floer, Symplectic fixed points and holomorphic spheres, Comm. Math. Phys., 120 (1989), 575-611.

[Fr1] J. Franks, Geodesics on $S^{2}$ and periodic points of annulus homeomorphisms, Invent. Math., 108 (1992), 403-418.

[Fr2] J. Franks, Area preserving homeomorphisms of open surfaces of genus zero, New York Jour. of Math., 2 (1996), 1-19.

[FH] J. Franks, M. Handel, Periodic points of Hamiltonian surface diffeomorphisms, Geom. Topol., 7 (2003), 713-756.

[GaL] D. Gatien, F. Lalonde, Holomorphic cylinders with Lagrangian boundaries and Hamiltonian dynamics, Duke Math. J., 102 (2000), 485-511.

[GhdlH] E. Ghys, P. de la Harpe, L'action d'un groupe hyperbolique sur son bord, in Sur les groupes hyperboliques d'aprés Mikhael Gromov (Bern, 1988), Progr. Math., 83, Birkhäuser Boston, 1990, pp. 79-102.

[Gi1] V.L. Ginzburg, Coisotropic intersections, Duke Math. J., 140 (2007), 111-163.

[Gi2] V.L. Ginzburg, The Conley conjecture, Ann. of Math., 172 (2010), 1127-1180.

[GG1] V.L. Ginzburg, B.Z. Gürel, Action and index spectra and periodic orbits in Hamiltonian dynamics, Geom. Topol., 13 (2009), 2745-2805.

[GG2] V.L. Ginzburg, B.Z. Gürel, Local Floer homology and the action gap, J. Sympl. Geom., 8 (2010), 323-357.

[GG3] V.L. Ginzburg, B.Z. Gürel, Conley conjecture for negative monotone symplectic manifolds, Int. Math. Res. Not. IMRN, 2011, doi:10.1093/imrn/rnr081.

[GG4] V.L. Ginzburg, B.Z. Gürel, Hyperbolic fixed points and periodic orbits of Hamiltonian diffeomorphisms, Preprint 2012, arXiv:1208.1733.

[Gü] B.Z. Gürel, Periodic orbits of Hamiltonian systems linear and hyperbolic at infinity, Preprint 2012, arXiv:1209.3529.

[He] D. Hein, The Conley conjecture for irrational symplectic manifolds, Preprint 2009, arXiv:0912.2064; to appear in J. Sympl. Geom.

[Hi1] N. Hingston, Subharmonic solutions of Hamiltonian equations on tori, Ann. of Math., 170 (2009), 525-560.

[Hi2] N. Hingston, On the growth of the number of closed geodesics on the two sphere, Internat. Math. Res. Notices (1993), no. 9, 253-262.

[HZ] H. Hofer, E. Zehnder, Symplectic Invariants and Hamiltonian Dynamics, Birkäuser, 1994.

[Ked] J. Kędra, Symplectically hyperbolic manifolds, Differential Geom. Appl., 27 (2009), 455-463.

[Ker] E. Kerman, On primes and period growth for Hamiltonian diffeomorphisms, J. Mod. Dyn., 6 (2012), 41-58.

[LeC] P. Le Calvez, Periodic orbits of Hamiltonian homeomorphisms of surfaces, Duke Math. J., 133 (2006), 125-184.

[Lee] Y-J. Lee, Non-contractible periodic orbits, Gromov invariants, and Floer-theoretic torsions, Preprint 2003, arXiv:math/0308185.

[Ma] G.A. Margulis, Applications of ergodic theory to the investigations of manifolds of negative curvature, Func. Anal. and Appl., 3 (1969), 335-336.

[Ni] C.J. Niche, Non-contractible periodic orbits of Hamiltonian flows on twisted cotangent bundles, Discrete Contin. Dyn. Syst., 14 (2006), 617-630.

[PS] R. Philips, P. Sarnak, Geodesics in homology classes, Duke Math. J., 55 (1987), 287297. 
[Sa] D.A. Salamon, Lectures on Floer homology, in Symplectic Geometry and Topology, Eds: Y. Eliashberg and L. Traynor, IAS/Park City Mathematics series, 7 (1999), pp. 143-230.

[SZ] D. Salamon, E. Zehnder, Morse theory for periodic solutions of Hamiltonian systems and the Maslov index, Comm. Pure Appl. Math., 45 (1992), 1303-1360.

[Vi] C. Viterbo, Symplectic topology as the geometry of generating functions, Math. Ann., 292 (1992), 685-710.

[We] J. Weber, Noncontractible periodic orbits in cotangent bundles and Floer homology, Duke Math. J., 133 (2006), 527-568.

BG: Department of Mathematics, Vanderbilt University, Nashville, TN 37240 , USA

E-mail address: basak.gurel@vanderbilt.edu 